\title{
REGIONALE INTERMANAGEMENT KOMMISSION: LOKALER BEREICH DER ÜBEREINSTIMMUNG UND INTERFÖDERATIVE ARTIKULATION?
}

\section{ORIGINALER ARTIKEL}

SANTOS, Nadja Romeiro dos ${ }^{1}$, COTA, Ana Lídia Soares²

SANTOS, Nadja Romeiro dos. COTA, Ana Lídia Soares. Regionale Intermanagement Kommission: Lokaler Bereich der Übereinstimmung und interföderative Artikulation? Revista Científica Multidisciplinar Núcleo do Conhecimento. Jahrgang 06, Ed. 09, Vol. 03, S. 173-189. September 2021. ISSN: 2448-0959, Zugangslink: https://www.nucleodoconhecimento.com.br/technologiede/regionale-intermanagement,

DOI: 10.32749/nucleodoconhecimento.com.br/technologie-de/regionale-intermanagement

\section{ZUSAMMENFASSUNG}

Die Regionale Intermanagement Kommission (CIR) gestaltet den Raum für Vereinbarung, Artikulation, Zusammenarbeit und Stärkung der Gesundheitsregion. Ziel dieser Studie war es, die normativen Bestimmungen zu reflektieren, die der Konstruktion der Kommission als Ort der Konsolidierung des Regionalmanagements zugrunde liegen, um interföderative Konflikte abzuschwächen. Als Leitfrage: Wie bieten die regionalen Intermanagementkommissionen lokale Räume der Übereinstimmung und interföderativen Artikulation? Qualitative Studie, basierend auf dokumentarischen Recherchen, basierend auf der Analyse der Resolutionen der regionalen Inter-Management-Kommissionen von Alagoas, entsprechend dem Zeitraum von 2017 bis 2019, unter Verwendung des Dekrets 7.508/11 als

\footnotetext{
${ }^{1}$ Doktorand im Graduiertenkolleg Gesellschaft, Technologien und öffentliche Ordnung. Universitätszentrum Tiradentes (UNIT).

2 Promotion in Kinderzahnheilkunde, Professor am Studiengang Zahnmedizin und am Graduiertenprogramm Gesellschaft, Technologie und öffentliche Politik (UNIT/AL) Universitätszentrum Tiradentes.
}

RC: 98473

Verfügbar in: https://www.nucleodoconhecimento.com.br/technologie-de/regionaleintermanagement 
Referenzstandard. Die Ergebnisse wurden in vier Kategorien eingeteilt: Regionale Gesundheitsplanung; Organisation von Maßnahmen und Dienstleistungen im Gesundheitswesen: Gesundheitsregion; Föderalismus, CIR und die Finanzierung von Gesundheitsdiensten; Governance und CIR: Verhandlungs- und Entscheidungskanal zwischen kommunalen Managern und dem Staat innerhalb der Gesundheitsregion. Die regionalen Intermanagementkommissionen entwickeln eine wichtige Arbeit in den Diskussionen über die Regionalpolitik, da sie ein grundlegendes Instrument zur Stärkung der Regierungsführung in den Gebieten sind, indem sie über die Zuweisung und Verteilung von Ressourcen verhandeln, die für die Planung, interföderative Pakte und die Gesundheitsfinanzierung unerlässlich sind und eine Schlüsselrolle für das regionale Gesundheitssystem von Alagoas spielen. demokratischer, politischer und kooperativer Raum.

Stichworte: Regionalisierung, Gesundheitsmanagement, Einheitliches Gesundheitssystem, Föderalismus, Gesundheitspolitik.

\section{EINLEITUNG}

Brasiliens Gesundheitspolitik wurde formuliert, um Artikel 196 der Bundesverfassung (CF/1988) zu entsprechen, basierend auf Maßnahmen und Dienstleistungen im Bereich der öffentlichen Gesundheit, die Teil eines regionalisierten und hierarchischen Netzwerks sind, das ein einziges System (SUS) darstellt, das nach verschiedenen Richtlinien organisiert ist, einschließlich Dezentralisierung, umfassender Versorgung und Beteiligung der Gemeinschaft (Artikel 198) (BRASIL, 1988).

Ein wichtiger Fortschritt in diesem Sinne war die Regulierung des BioGesundheitsgesetzes (Gesetz 8.080/90) durch das im Juli 2011 veröffentlichte Bundesbeschluss Nr. 7.508, der den regulatorischen Rahmen zur Verdichtung der verfassungsmäßigen und gesetzlichen Bestimmungen im Zusammenhang mit der Verwirklichung des Grundrechts auf Gesundheit in Brasilien, Nodits in Bezug auf die

RC: 98473

Verfügbar in: https://www.nucleodoconhecimento.com.br/technologie-de/regionaleintermanagement 
Dezentralisierung der öffentlichen Gesundheit, mit der Organisation des SUS, der Planung von Gesundheitsmaßnahmen und interföderativen Artikulation.

Eine der wichtigsten Errungenschaften bezieht sich auf die Operationalisierung der SUS innerhalb des regionalen Territoriums, von der Validierung der Räume der Vereinbarung und Beratung, mit Blick auf die Definition von Regeln für die geteilte Verwaltung, genannt Regional Inter-Management Commissions (CIR), wobei der Prozess der Dezentralisierung der öffentlichen Gesundheit, eine der verfassungsmäßigen Prämissen, mit der Legitimation regionaler Vereinbarungsräume intensiviert wird.

Dieser politisch-administrative Prozess der SUS in Brasilien, ein siegreiches Modell in der Verfassungsgebenden Nationalversammlung (1986-1988), war die Konsektiere einer der Hauptforderungen der Bewegung für Gesundheitsreform, die aufgrund der verschiedenen Kritiken an dem damals im Land geltenden Zentralisierungsmodell aufgebaut wurde und damals vom Nationalen Institut für medizinische Hilfe der sozialen Sicherheit (INAMPS) ausgeführt wurde.

Dieses Organisationsprinzip wurde auch in die anderen eingeführten öffentlichen Politiken einbezogen, insbesondere in den Bereichen Gesundheit, Bildung und Sozialhilfe, die in gegenseitiger Artikulation die Grundlagen eines Staates des sozialen Schutzes strukturieren.

Nach Wagner (2006) neigt die Dezentralisierung dazu, eine Entscheidungsautonomie in Bezug auf die Gesundheit für föderierte Entitäten zu erzeugen. Die zunehmende finanzielle Zentralisierung des Staates, zusammen mit der fortgesetzten Ausübung der fiskalischen Regulierung von Staaten und Gemeinden und einer entscheidenden Rolle bei der Politikformulierung, führte zu einer Kombination aus staatlicher Zentralisierung und Dezentralisierung sektoraler Politiken, die im Falle der Gesundheit immer von der Einführung durch den Bund abhängig waren (VIANA et al., 2002).

RC: 98473

Verfügbar in: https://www.nucleodoconhecimento.com.br/technologie-de/regionaleintermanagement 
Die Region wird als der kontinuierliche geografische Raum verstanden, der aus Clustern benachbarter Gemeinden besteht, die aus der Existenz kultureller, sozialer und wirtschaftlicher Identitäten und anderer definiert sind und darauf abzielen, Gesundheitsmaßnahmen und -dienstleistungen zu integrieren, zu organisieren, zu planen und durchzuführen (BRASIL, 2011).

In diesem Umfeld zeichnen sich die CIRs als Instrumente zur Unterstützung der Umsetzung von Gesundheitspolitiken in föderativen Regionen aus. In der Praxis fungieren sie als Instanzen der regionalen Governance von Gesundheitsnetzwerken, deren Handeln relevant ist, um die Dynamik der Regionalisierung zu bewirken, Probleme zu identifizieren, Prioritäten $z u$ definieren und Lösungen für ihre Organisation vorzuschlagen, die bei der Konstitution gemeinsamer Ziele und bei der Etablierung einer Logik helfen, die sich auf das Wohlergehen der Bevölkerung und die Bereitstellung von Gesundheitsdienstleistungen konzentriert.

Im Zusammenhang mit der Aufrechterhaltung des kommunalen Protagonismus und der Stärkung der Beziehungen der Interdependenz und der sanitären Mitverantwortung zwischen dem Staat und den kommunalen Einrichtungen entsteht der CIR, der die Verwaltung der regionalen Gesundheitspolitik auf der Grundlage der Kenntnis der epidemiologischen und sozioökonomischen Realität der Benachbarten Gemeinden als Auftrag hat.

In Bezug auf den Zustand von Alagoas koordiniert der CIR den Aufbau des Regionales Pflegenetzwerk (RAR), das den Zugang der Bevölkerung zu Gesundheitsdiensten sicherstellt, die die für die in ihrem Gebiet eingesetzten Gemeinden festgelegten Ziele regulieren, überwachen und bewerten.

Daher zielt dieser Artikel darauf ab, über die normativen Bestimmungen nachzudenken, die der Konstruktion von CIR als Räume für die Konsolidierung des regionalen Managements zugrunde liegen, die interföderative Konflikte abschwächen, die Regionalisierung in den SUS analysieren und versuchen, den

RC: 98473

Verfügbar in: https://www.nucleodoconhecimento.com.br/technologie-de/regionaleintermanagement 
Einfluss der zwischenstaatlichen Zusammenarbeit auf die Stärkung dieser Strategie zu überprüfen. Um die vorgeschlagene Analyse zu unterstützen, wurden theoretische Referenzen zum Thema überprüft, beginnend mit dem Überblick über die Grundlagen des Föderalismus, die SUS in der brasilianischen föderativen Struktur und die Konzepte der Dezentralisierung und Regionalisierung, die mit der Diskussion einiger kritischer Faktoren endeten. Angesichts der obigen Überlegungen stellte sich folgende Frage: Wie stellt sich das CIR lokale Räume der Übereinstimmung und interföderativen Artikulation?

\section{METHODIK}

Dies ist eine deskriptive und explorative Studie mit einem qualitativen Ansatz, die durch eine thematische Überprüfung und Analyse des Inhalts der offiziellen Dokumente des CIR / AL als Raum für die Vereinbarung und interföderative Artikulation des SUS durchgeführt wird, die es ermöglicht, Wissen über das untersuchte Objekt zu aggregieren und auch den Fortschritt der öffentlichen Gesundheitspolitik aus dem Regionalisierungsprozess zu analysieren (MINAYO, 2010).

Wir analysierten die vom CIR ausgearbeiteten Resolutionen für den Zeitraum 2017 bis 2019, die der Öffentlichkeit auf der Seite des Amtsblatts des Staates Alagoas (DOE/ AL) http://www.imprensaoficial.com.br/diario-oficial zur Verfügung stehen, wobei wir das Dekret 7.508/11 als Referenzstandard verwendeten. Die Ergebnisse wurden in vier analytische Kategorien systematisiert, gruppiert nach dem Lesen der Dokumente, deren Ergebnisse in Tabelle 1 angeordnet sind, das heißt: Planung (Kategorie 1); Organisation (Kategorie 2); (Kategorie 3) und Governance (Kategorie 4).

RC: 98473

Verfügbar in: https://www.nucleodoconhecimento.com.br/technologie-de/regionaleintermanagement 


\section{ERGEBNISSE}

Von den insgesamt 192 gelesenen Resolutionen wurde ein analytischer Rahmen erstellt (Tabelle 1), der die Hauptberatungen des CIR und seine jeweilige Bedeutung als relevante Inhalte der zuvor gewählten Kategorien als Leitlinie der Diskussion anordnete.

Tabelle I: Charakterisierung der Resolutionen nach Analysekategorien für den Zeitraum 2017-2019.

\begin{tabular}{|c|c|c|}
\hline Kategorie & Sinn für Überlegung & Wichtigste Beratungen \\
\hline \multirow[t]{5}{*}{ Planung } & \multirow{5}{*}{$\begin{array}{l}\text { Kooperationsstrategien, } \\
\text { die zum Abkommen } \\
\text { beitragen und den } \\
\text { Zugang zu } \\
\text { Bevölkerung } \\
\text { Gesundheitsdiensten } \\
\text { gewährleisten. } \\
\text { Strategie, die Qualität } \\
\text { bei der Umsetzung von } \\
\text { Gesundheitspolitiken } \\
\text { bevorzugt. }\end{array}$} & $\begin{array}{l}\text { Genehmigt die von den technischen } \\
\text { Bereichen des Gesundheitsministerium - } \\
\text { SESAU vorgeschlagenen Ziele unter } \\
\text { Bezugnahme auf interföderative } \\
\text { Indikatoren für den Zeitraum 2017-2021 }\end{array}$ \\
\hline & & $\begin{array}{l}\text { Genehmigt Gesundheitsplanung, } \\
\text { Gesundheitsversorgung und } \\
\text { interföderative Artikulation }\end{array}$ \\
\hline & & $\begin{array}{l}\text { Genehmigt den staatlichen Plan zur } \\
\text { Humanisierung der Gesundheit des } \\
\text { Staates Alagoas, Vierjahresplan } 2016 \text { - } \\
2019 \text { und den Betriebsplan } 2017\end{array}$ \\
\hline & & $\begin{array}{l}\text { Reagiert auf die Ziele der Interföderativen } \\
\text { Indikatoren. }\end{array}$ \\
\hline & & $\begin{array}{l}\text { Ändert den staatlichen Plan des } \\
\text { Pflegenetzes für Menschen mit } \\
\text { Behinderungen, um einen finanziellen } \\
\text { Investitionsanreiz des } \\
\text { Gesundheitsministeriums einzufügen }\end{array}$ \\
\hline
\end{tabular}

$\mathrm{RC}: 98473$

Verfügbar in: https://www.nucleodoconhecimento.com.br/technologie-de/regionaleintermanagement 


\begin{tabular}{|c|c|c|}
\hline & & $\begin{array}{l}\text { Genehmigt den Dienstaktionsplan als } \\
\text { Referenz für die Makroregion } \\
\text { "Gesundheit" }\end{array}$ \\
\hline \multirow[t]{10}{*}{ Organisation } & \multirow{10}{*}{$\begin{array}{l}\text { Organisation von } \\
\text { Dienstleistungen, die } \\
\text { die Stärkung des } \\
\text { Gesundheitsnetzwerks } \\
\text { in der Region fördern. }\end{array}$} & Genehmigt die Dienstaktivierung \\
\hline & & $\begin{array}{l}\text { Genehmigt die Verknüpfungskarte von } \\
\text { Laborreferenzen für die Durchführung } \\
\text { zytopathologischer Tests für das } \\
\text { Gebärmutterhalskrebs-Screening. }\end{array}$ \\
\hline & & $\begin{array}{l}\text { Genehmigt das Handbuch der } \\
\text { Behandlung außer haus - TFD Alagoas. }\end{array}$ \\
\hline & & $\begin{array}{l}\text { Genehmigt die Implementierung von } \\
\text { Intensivbetten innerhalb des Notfall- und } \\
\text { Notfallnetzwerks (RUE) }\end{array}$ \\
\hline & & $\begin{array}{l}\text { Genehmigt Umverteilung von Betten für } \\
\text { psychische Gesundheit in der } \\
\text { Gesundheitsregion }\end{array}$ \\
\hline & & $\begin{array}{l}\text { Genehmigt das Einsetzen der } \\
\text { Notaufnahmeeinheit der Größe III }\end{array}$ \\
\hline & & $\begin{array}{l}\text { Genehmigt die Implementierung der } \\
\text { Langzeitpflegestation - UCP }\end{array}$ \\
\hline & & $\begin{array}{l}\text { die Qualifizierung der hinteren Betten des } \\
\text { Notfall- und Notfallnetzes (RUE) } \\
\text { genehmigt, }\end{array}$ \\
\hline & & $\begin{array}{l}\text { Genehmigt die Neuqualifizierung des } \\
\text { Dienstes }\end{array}$ \\
\hline & & $\begin{array}{l}\text { Genehmigt die Einrichtung eines } \\
\text { Notfallzentrums für Patienten mit } \\
\text { Schlaganfall, Typ III, in einem } \\
\text { Gesundheitsdienst als Referenz für die }\end{array}$ \\
\hline
\end{tabular}

RC: 98473

Verfügbar in: https://www.nucleodoconhecimento.com.br/technologie-de/regionaleintermanagement 


\begin{tabular}{|c|c|c|}
\hline & & $\begin{array}{l}\text { Gesundheitsregion } \\
\text { Genehmigt die Änderung der Spezialität } \\
\text { des Eingangstürdienstes der klinischen } \\
\text { Dringlichkeit. }\end{array}$ \\
\hline \multirow[t]{5}{*}{ Finanzierung } & \multirow{5}{*}{$\begin{array}{l}\text { Strategie } \\
\text { Finanzierung und } \\
\text { Förderung der } \\
\text { Regionalisierung. } \\
\text { Umsetzung der } \\
\text { öffentlichen Politik in } \\
\text { der Region. }\end{array}$} & $\begin{array}{l}\text { Billigt die Verlagerung der physischen } \\
\text { und finanziellen Obergrenzen in die } \\
\text { vereinbarte und integrierte } \\
\text { Programmplanung }\end{array}$ \\
\hline & & $\begin{array}{lccc}\text { Finanzierung und } & \text { Transfer } & \text { von } \\
\text { Bundesmitteln } & - & \text { Notfall- } & \text { und } \\
\text { Notfallversorgungsnetz } & & \end{array}$ \\
\hline & & $\begin{array}{l}\text { Genehmigt die Neuzuweisung von } \\
\text { Finanzmitteln für die Übertragung von } \\
\text { SUS aus dem Kostenblock für öffentliche } \\
\text { Gesundheitsmaßnahmen und -dienste } \\
\text { der Gemeinde unter Bezugnahme auf die } \\
\text { Ressourcen der materiellen und } \\
\text { finanziellen Obergrenzen gemäß der } \\
\text { vereinbarten und integrierten } \\
\text { Programmplanung für die Pflege mittlerer } \\
\text { und hoher Komplexität ( Ambulante und } \\
\text { Krankenhaus-MAC) }\end{array}$ \\
\hline & & $\begin{array}{l}\text { Genehmigt den Antrag auf Erhöhung der } \\
\text { finanziellen Mittel der mittleren und hohen } \\
\text { ambulanten und Krankenhauskomplexität } \\
\text { (MAC) der Gemeinde. }\end{array}$ \\
\hline & & $\begin{array}{l}\text { Strategien und Kompensation - FAEC für } \\
\text { die jährliche Finanzgrenzkomponente der } \\
\text { ambulanten und stationären Versorgung }\end{array}$ \\
\hline
\end{tabular}

RC: 98473

Verfügbar in: https://www.nucleodoconhecimento.com.br/technologie-de/regionaleintermanagement 


\begin{tabular}{|c|c|c|}
\hline & & $\begin{array}{l}\text { mittlerer und hoher Komplexität - MAC } \\
\text { der Länder, des Bundesbezirks und der } \\
\text { Gemeinden; } \\
\text { Genehmigt finanzielle Grenzen, die der } \\
\text { Gemeinde zur Verfügung gestellt werden } \\
\text { sollen, um elektive chirurgische Eingriffe } \\
\text { zu finanzieren, die sich auf die } \\
\text { Gesundheitsregion beziehen. }\end{array}$ \\
\hline Governance & $\begin{array}{l}\text { Regierungsstrategien } \\
\text { zur Stärkung der } \\
\text { regionalen Governance } \\
\text { und zur Verbesserung } \\
\text { der } \\
\text { Kommunikationsnetze, } \\
\text { die zur Integralität } \\
\text { föderativer } \\
\text { Maßnahmen beitragen. } \\
\text { Unterstützung der } \\
\text { geteilten } \\
\text { Mittelverwaltung. }\end{array}$ & $\begin{array}{l}\text { Genehmigt die Kommission der } \\
\text { Technischen Kammer der regionalen } \\
\text { Intermanager } \\
\begin{array}{l}\text { Genehmigt den } \\
\text { regionalen }\end{array} \\
\begin{array}{l}\text { Ausschusses. } \\
\text { Inter-Management- }\end{array}\end{array}$ \\
\hline
\end{tabular}

Quelle: Eigene Autorenschaft, 2020

\section{DISKUSSION}

\subsection{REGIONALE GESUNDHEITSPLANUNG}

Planung ist ein permanenter und dynamischer Prozess und sollte auch kurzfristig betrachtete Notfallsituationen einbeziehen. Im Gesundheitsbereich ist die Planung die Zuständigkeit der drei Regierungsbereiche und bildet die Grundlage für die Durchführung, Überwachung, Bewertung, Kontrolle und Verwaltung des

RC: 98473

Verfügbar in: https://www.nucleodoconhecimento.com.br/technologie-de/regionaleintermanagement 
Gesundheitssystems unter der Annahme der Kenntnis der bestehenden Realität und der verfügbaren und notwendigen Instrumente, um die vorgeschlagenen Ziele zu erreichen.

Laut Santos (2017) schadet das Fehlen von Planung der Organisation der Gesundheitsdienste, schwächt die Zusammenarbeit und Koordination der Regionalisierungspolitik. Tatsächlich legt die Bundesverfassung in ihrem Art. 176 fest, dass es sich um eine obligatorische Planung für die öffentliche Macht und einen Anreiz für den privaten Sektor handelt (BRASIL, 1988).

Der Prozess der Regionalisierung der Gesundheit ermöglicht die Zuweisung von Dienstleistungen im Gesundheitsnetzwerk, um alle Ebenen der Gesundheit entsprechend den Bedürfnissen des Einzelnen zu betrachten. Die in dieser Studie analysierten Dokumente zeigten, dass Planung zu einer wichtigen Agenda in den Diskussionsagenden von Managern in Gesundheitsregionen geworden ist.

Eine der Folgen der Planung besteht darin, die Verantwortlichkeiten der drei Regierungsbereiche, die Definition der Bereitstellung und Verwaltung von Dienstleistungen und die Bereitstellung finanzieller Ressourcen zu klären. Auf diese Weise muss der Planungs- und Budgetierungsprozess unteilbar sein. Daher ist die Haushaltsplanung im Gesundheitswesen als integraler Bestandteil und Detail der Gesundheitspläne von wesentlicher Bedeutung (BRASIL, 2011).

Laut Duarte und anderen Mitarbeitern (2018) steigt die Organisation der Planung und des Budgets der SUS auf, integriert auf regionalisierte Weise, von der Anerkennung der Gesundheitsprobleme jeder Gemeinde, die durch die Artikulation zwischen autonomen Einheiten aufgebaut wird, um die Lebensqualität der Bevölkerung durch die Organisation von Gesundheitsdiensten zu gewährleisten. In der Praxis wird die Planung und Organisation der Aktivitäten jeder Leitungsebene des SUS durch die Organisation des Gesundheitsplans in jeder Jurisdiktion, in der seine Finanzierung

RC: 98473

Verfügbar in: https://www.nucleodoconhecimento.com.br/technologie-de/regionaleintermanagement 
im Haushaltsvorschlag vorgesehen ist (BRASIL, 1990), von den gesundheitlichen Bedürfnissen der Bevölkerung geleitet.

Die integrierte Raumordnung etabliert den CIR als einen Raum für Artikulation, Verhandlung und Vereinbarung zwischen den unterzeichnenden Einheiten, der die Identifizierung, Definition von Prioritäten und Verhandlung von Lösungen für die Organisation eines regionalen Netzwerks von Maßnahmen und Dienstleistungen der integrierten und resolutiven Gesundheitsversorgung nach den Prinzipien des SUS ermöglicht (BRASIL, 2011).

Der CIR hat die Bedeutung der Konsolidierung der Politik im Bereich der öffentlichen Gesundheit durch die Überwachung der integrierten vereinbarten Programmplanung, um die Konstruktion des Regulierungsdesigns mit Der Definition von Strömen und Protokollen im Hinblick auf die Qualifizierung der sozialen Kontrolle und die Organisation der geteilten Mittelverwaltung in aufsteigender Weise zu begünstigen, geplant mit der Definition der finanziellen und administrativen Ressourcen, die zur Regionalisierung beitragen (BRASIL, 2011).

Die Ergebnisse der Dokumentenanalyse zeigen, dass das CIR ein Raum mit dem Potenzial ist, institutionelle Kapazitäten für die regionale Raumplanung und koordinierung zu entwickeln, die darauf abzielen, Unternehmensinteressen zu überwinden und eine lokale Governance auf der Grundlage der gemeinsamen Verwaltung und Demokratisierung von Entscheidungen und zwischenstaatlicher Zusammenarbeit zu schaffen.

\subsection{ORGANISATION VON MABNAHMEN UND DIENSTLEISTUNGEN IM GESUNDHEITSWESEN: GESUNDHEITSREGION}

Die Analyse der Resolutionen des CIR zeigt, dass die Organisation der Gesundheitsversorgung eine ständige "Phase" der Diskussion in den föderativen

RC: 98473

Verfügbar in: https://www.nucleodoconhecimento.com.br/technologie-de/regionaleintermanagement 
Bereichen ist, was zeigt, dass Gesundheitsnetzwerke die Richtung für die Arbeit der Vereinbarung innerhalb der Inter-Management-Kommissionen sind (BRASIL, 2011).

Die Organisation der Gesundheitsdienste sollte in den Gesundheitsregionen in Betracht gezogen werden, die einen kontinuierlichen geografischen Raum bilden, der von einem Cluster von Gemeinden integriert wird, der als territoriale Grundlage für die Planung der Gesundheitsversorgung gebildet wird, die sich aus der gemeinsamen Region der regionalen Finanzierung durch die Ausführung von Dienstleistungen ergibt, die auf die Governance in der Region abzielen, durch Zusammenarbeit zwischen föderativen Einheiten; sollte Maßnahmen der Primärversorgung, der psychosozialen Versorgung, der Gesundheitsüberwachung, des Notfalls, der spezialisierten ambulanten und krankenhausmäßigen Versorgung enthalten (BRASIL, 2011).

Für die Integration von Maßnahmen und Diensten im Bereich der öffentlichen Gesundheit in ein regionalisiertes Netzwerk ist es notwendig, dass die föderierten Einheiten auf integrierte Weise arbeiten.

Diese Aggregation von Dienstleistungen in der Region ist notwendig, um die Integralität der Pflege durch den Fluss von Referenz und Gegenreferenz zu gewährleisten, und es ist dem größeren Manager überlassen, in der Region für Dienstleistungen von größerer Komplexität zu antworten, die Skalierung und andere administrative und technologische Komponenten erfordern, auf die Gemeinden anderer föderierter Einheiten zugreifen können (ANDRADE; FRANCISCHETTI, 2019).

Laut Santos (2017) ist die Zusammenarbeit zwischen den Instanzen der Vereinbarung im Territorium als eine Möglichkeit, die Betriebsräume des SUS zu organisieren, ein Schlüsselpunkt, da seine sanitäre, technologische und finanzielle Singularität für die Strukturierung des SUS von grundlegender Bedeutung ist. Ohne

RC: 98473

Verfügbar in: https://www.nucleodoconhecimento.com.br/technologie-de/regionaleintermanagement 
dieses Organisationsformat war das Gesundheitssystem nicht in der Lage, eine umfassende, gleichberechtigte und dezentrale Versorgung zu bieten.

Um dieses Netz von Dienstleistungen innerhalb des Hoheitsgebiets zu organisieren und zu integrieren, übernehmen die Unterzeichner die im Organisationsvertrag für öffentliche Maßnahmen (COAP) festgelegte Verantwortung mit dem Ziel, Gesundheitsmaßnahmen und -dienste regionalisiert und hierarchisch zu gewährleisten, wobei die Definition von Exekutive, Haushalt, Finanzen und Kontrolle der Evaluierung zwischen den Staaten definiert ist. Gemeinde und Union, die Gesundheitsziele für Kosten und Investitionen festlegen, die der Bevölkerung eine vollständige Versorgung garantieren (BRASIL, 1990, 2010, 2011; SANTOS, 2017).

Die Entscheidung, die Gesundheitsversorgung und das Management zu qualifizieren, beinhaltet technische, ethische und kulturelle Aspekte, mit der Notwendigkeit, den kooperativen politischen Pakt zwischen den Leitungsorganen des Systems einzuhalten, der durch eine "feine Verbindung von Technik und Politik" ausgedrückt wird, um die für den Wandel erforderlichen Investitionen und Ressourcen sicherzustellen (BRASIL, 2010, 2011).

Die Lösung besteht darin, den Prozess der Organisation des Gesundheitssystems zu erneuern und Maßnahmen und Dienstleistungen bei der Entwicklung regionalisierter Gesundheitsnetzwerke (RAS) neu zu ordnen, um einen positiven Einfluss auf die Gesundheitsindikatoren der Bevölkerung zu erzielen.

Der SUS-Managementpakt legt die Notwendigkeit fest, RAS zu entwerfen, die in den Gebieten der Gesundheitsregionen organisiert sind, was der privilegierte Raum für den Aufbau vereinbarter Verantwortlichkeiten ist und die Integration von Richtlinien und Programmen durch die gemeinsame Aktion der Bundes-, Landes- und Kommunalebene ermöglicht (BRASIL, 2010).

RC: 98473

Verfügbar in: https://www.nucleodoconhecimento.com.br/technologie-de/regionaleintermanagement 
Das Dekret 7508/11 in Kapitel II legt fest, dass die SUS durch ein regionalisiertes und hierarchisches Netzwerk gebildet werden müssen und dass ihre Gesundheitsregionen so organisiert sein sollten, dass sie Gesundheitsdienstleistungen anbieten, die auf einem Niveau zunehmender Komplexität artikuliert sind, um eine umfassende Versorgung und Gesundheitsversorgung zu gewährleisten (BRASIL, 2001).

Der Pakt etablierte den regionalen Raum als privilegierten Ort für den Aufbau der vereinbarten Verantwortlichkeiten, die die Erfüllung der übernommenen Verantwortlichkeiten und vereinbarten Ziele erfordern, und jeder Regierungsbereich ist mitverantwortlich für die Verwaltung der Politiken mit ausdrücklichen Verantwortlichkeiten (BRASIL, 2006).

Die Ergebnisse zeigen deutlich die Organisation der Gesundheitsdienste durch Versorgungsnetzwerke und föderative Vereinbarungen zur Ausführung von Dienstleistungen, die die Bedürfnisse der Gesundheitsregionen berücksichtigt. Laut Santos (2017) ist es notwendig, auf integrierte und systemische Weise zu handeln und die Kompetenz flexibel zu teilen, um eine Einigung zwischen föderativen Einheiten zu gewährleisten, die die Gesundheit der Bevölkerung beeinflussen, die Intersektoralität fördern und soziale Ungleichheiten abbauen können.

\subsection{FÖDERALISMUS, VERWALTUNGSKOMMISSIONEN UND FINANZIERUNG VON GESUNDHEITSDIENSTEN}

Föderalismus ist eine Organisationsform des Staates, die aus dem dialektischen Gleichgewicht zwischen Zentralisierung und Dezentralisierung der politischen Macht geboren wurde, entsprechend der Notwendigkeit, die Einheit in der Vielfalt aufrechtzuerhalten, ohne die Macht in einem einzigen Kern zu konzentrieren oder zu pulverisieren (DOURADO; ELIAS, 2011).

$\mathrm{RC}: 98473$

Verfügbar in: https://www.nucleodoconhecimento.com.br/technologie-de/regionaleintermanagement 
Laut Curvina (2017) entstand der Föderalismus 1787 mit der Verfassung der Vereinigten Staaten von Amerika. Laut dem Autor wurde das Modell des amerikanischen Föderalismus von den anderen Ländern kopiert und konvergierte ganz oder teilweise zu einer Dualität von Kompetenzen, die durch die Koexistenz von mindestens zwei konstitutionellen Sphären autonomer Macht gekennzeichnet ist, die auf demselben Territorium und derselben Bevölkerung handeln (MENICUCCI, 2019).

In Brasilien begann der Föderalismus mit der Existenz einer Einheit mit zentralisierter Macht, die auf mehrere Einheiten (föderierte Einheiten) verteilt wurde, ohne die zentrale Macht zu eliminieren. Diese an Länder und Gemeinden delegierte Befugnis wird durch Bundes-, Landes- und Kommunalgesetzgebung verfassungsrechtlich geregelt. Ein System des kooperativen Föderalismus, sowohl für wirtschaftliche, soziale und kulturelle Fragen, durch Programme und gemeinsame Finanzierung, die von Viana et al (2008) betrachtet wurden. "institutionelle Flexibilität", die eine zwischenstaatliche Zusammenarbeit anstrebt, um gemeinsame Ziele zu erreichen.

Barata (2004) es sollte angemerkt werden, dass es keine Hierarchie in der föderalen Organisation gibt, weil jede Machtsphäre einer bestimmten Kompetenz entspricht, die Autonomie der föderierten Entitäten vorausspricht. Obwohl das Studium des Föderalismus die Untersuchung der Besonderheiten jeder Föderation durchläuft, gibt es gemeinsame Merkmale, die die Grundlagen des Bundesstaates darstellen.

Im gegenwärtigen historischen Kontext, in dem die Nationalstaaten die vorherrschende Rolle bei der Förderung des sozialen Wohlergehens spielen, besteht die Notwendigkeit einer gegenseitigen Zusammenarbeit zwischen den Regierungsbereichen, um soziale und wirtschaftliche Ziele zu erreichen, die sich aus der Feststellung ergeben, dass die Ausübung bestimmter öffentlicher Funktionen keine ausschließliche oder hegemoniale Zuschreibung einer der föderierten Einheiten sein kann. Aufteilung der Zuständigkeiten, Zusammenarbeit und gemeinsame Interessen. Dies ist das heute vorherrschende Modell, das als kooperativer Föderalismus bezeichnet wird (CURVINA, 2017).

RC: 98473

Verfügbar in: https://www.nucleodoconhecimento.com.br/technologie-de/regionaleintermanagement 
In der Tat ist das Management der öffentlichen Politik von grundlegender Bedeutung für die Entwicklung von Zielen und Maßnahmen im sozioökonomischen Bereich, die auf das kollektive Wohlergehen der Bevölkerung abzielen, durch Zusammenarbeit in den drei Bereichen der Regierung durch die Konstruktion von Entscheidungsprozessen und geplanten Prozessen (SOARES, 2018). Diese Prozesse stellen das "inhärente Dilemma zwischen den direkten Verhandlungen der lokalen Regierungen und den von den zentralen Ideen geförderten Anreizen" dar, das sich aus wiederkehrenden Problemen ergibt, die durch Dezentralisierung und Zentralisierung dargestellt werden (DOURADO; ELIAS, 2011, S. 206).

Eine permanente Unterstützung des Sus-Managements ist die Stärkung der interföderativen Bindungen, die für die Konsolidierung des Systems notwendig sind. Das Organgesundheitsgesetz (Gesetz 8.080/90) legte neue Strategien und Instrumente für die Konsolidierung von Gesundheitsnetzwerken fest, bewertete den Aufbau von Kooperationsbeziehungen zwischen föderierten Einheiten durch COAP und stärkte die koordinierende Rolle von Staatsmanagern und Inter-ManagementKomitees (BRASIL, 1990).

In der Tat zielt die Dezentralisierung darauf ab, zwischenstaatliche Beziehungen bei der Umsetzung der öffentlichen Politik aufzubauen und die Bedeutung des Dialogs zwischen subnationalen Regierungen und der nationalen Regierung bei der Durchführung der Gesundheitspolitik zu stärken. Neben signifikanten Veränderungen im institutionellen Design in der Regierungsorganisation und im sozialen Bereich mit Schwerpunkt auf Kommunikation und Verantwortungsteilung zwischen föderalen Stellen im politischen und finanziellen Management der lokalen Gesundheitssysteme, Ausweitung der Abdeckung und Begünstigung des Zugangs der Bevölkerung zu Gesundheitsdiensten und -aktionen (MOREIRA; FERRÉ; ANDRADE, 2017).

Es ist unbestreitbar, dass die Dezentralisierung der finanziellen und Managementasymmetrie der Regierungsebenen, bei der Organisation und

RC: 98473

Verfügbar in: https://www.nucleodoconhecimento.com.br/technologie-de/regionaleintermanagement 
Verwaltung des heterogenen und nicht-institutionell integrierten Dienstleistungsnetzes mit Auswirkungen, die sich aus der Entstehung verschiedener lokaler und isolierter Systeme von Gesundheitsdiensten ergeben, sowie in der Fragmentierung und Desorganisation von Dienstleistungen, die der Gemeinschaft zur Verfügung gestellt werden,(LEVCOVITZ et al., 2001; SANTOS et al., 2015). Ein weiterer wichtiger Punkt, der durch die von den Autoren diskutierte Dezentralisierung verursacht wird, bezieht sich auf die Distanzierung von Regierungsbehörden, die für die Gesundheitsfinanzierung verantwortlich sind (PINAFO et al., 2020).

Die Beziehung zwischen Dezentralisierung, Effizienz und Gerechtigkeit ist von komplexen kausalen Netzen durchdrungen, jedoch deuten vorhandene Beweise darauf hin, dass in einem kontinentalen Land wie Brasilien, das aus räumlicher Vielfalt, territorialen Ungleichheiten, Verteilung, Vermittlung und Kontrollkosten besteht, die von der zentralisierten Verwaltung (AFFONSO, 1985; LIMA et al., 2015).

In der Tat, die geografische Konzentration von Dienstleistungen von größerer Komplizenschaft, die regionalen Unterschiede zwischen den Bevölkerungen und politisch-institutionellen Bedingungen der föderativen Einheiten in Brasilien, es besteht ein Bedarf und eine Nutzung der verfügbaren Ressourcen, mit einer Aufteilung der Verantwortung zwischen den Managementbereichen, um widersprüchliche Interessen verschiedener Regionen in Einklang zu bringen, mit dem Ziel, ein kooperatives und finanzielles Modell bei der Bereitstellung von Gesundheitsdienstleistungen zu schaffen (LIMA et al. 2012).

$\mathrm{RC}: 98473$

Verfügbar in: https://www.nucleodoconhecimento.com.br/technologie-de/regionaleintermanagement 
INTERMANAGEMENTKOMMISSIONEN - CIR: VERHANDLUNGSUND ENTSCHEIDUNGSKANAL ZWISCHEN KOMMUNALEN MANAGERN UND DEM STAAT INNERHALB DER GESUNDHEITSREGION

Die CIR/AL wurden am 22. August 2011 gegründet und ihre Regeln definieren sie als privilegierte Fälle von Verhandlungen, Artikulation, Vorschlägen und Vereinbarungen in Bezug auf die operativen Aspekte der SUS in der Gesundheitsregion unter Beachtung der Definitionen der Zweiseitigen Intermanagerkommission (CIB), die einen ständigen Raum für Solidarität und kooperatives Co-Management auf regionaler Ebene darstellt. von der Identifizierung eines Netzwerks organisierter, integrierter und resolutiver Gesundheitsdienste, die die Verbesserung und Anwendung der Prinzipien der SUS sicherstellen (COSEMS/AL, 2014).

Sie setzen sich aus den kommunalen Gesundheitsministern und der Vertretung des Staatssekretärs für Gesundheit zusammen, der das Vorrecht hat, den Einigungsprozess zu koordinieren, und ihre Entscheidungen werden im Konsens gemäß den Bestimmungen des Gesundheitspakts getroffen.

Im Bundesstaat Alagoas wurde durch die Genehmigung des RegionalisierungsMasterplans (PDR) das sanitäre territoriale Design neu definiert, in zwei Gesundheitsmakroregionen reorganisiert, die in zehn Sanitärregionen unterteilt sind, 06 (sechs) in der 1. Makroregion mit Sitz in Maceió und 04 (vier) in der 2. Makroregion mit Sitz in der Gemeinde Arapiraca.

Mit der Aufgabe, die regionale Gesundheitspolitik auf der Grundlage der Kenntnis der epidemiologischen und sozioökonomischen Realität der Gemeinde, des Territoriums und der Region zu verwalten, koordiniert das CIR den Bau des RAR, stellt den

RC: 98473

Verfügbar in: https://www.nucleodoconhecimento.com.br/technologie-de/regionaleintermanagement 
Zugang der Bevölkerung zu Gesundheitsdiensten sicher, reguliert, überwacht und bewertet die für die Gemeinden festgelegten Ziele.

Mit dem Ziel, den kooperativen Charakter der durch den Pakt für Gesundheit geschaffenen zwischenstaatlichen Beziehungen zu vertiefen und im Rahmen des SUS-Managements eine Reihe von Management- und Pflegeinstrumenten zu institutionalisieren, bringt das Dekret 7.508/2011 die Definition von Gesundheitsregionen und Gesundheitsnetzen, erstellt die Gesundheitskarte, die Nationale Liste der Gesundheitsmaßnahmen und -dienste - RENASES die Nationale Liste der unentbehrlichen Arzneimittel - RENAME, und COAP (BRASIL, 2011). Es legt die Verhandlungs- und zwischenstaatlichen Artikulationsgremien fest, die auf verschiedenen territorialen Skalen (national, staatlich und regional) tätig sind.

Nach der Habermas'schen Theorie braucht die Wirksamkeit wahrgenommener Forderungen im öffentlichen Raum Kommunikation und Dialogflüsse, das heißt ein deliberatives Demokratiemodell. In der republikanischen Konzeption sind der öffentliche und politische Raum und die Zivilgesellschaft als ihre Infrastruktur strategische Punkte demokratischer und kommunikativer Organisation. Die Funktion, die integrative Kraft und die demokratische Autonomie der Praxis der Verständigung zwischen den Subjekten zu gewährleisten (HABERMAS, 1995).

Unter strategischen Bedingungen treffen Individuen Entscheidungen, die die Konsequenzen ihres Handelns antizipieren, wählen politische Institutionen, mit einem Wort, handeln politisch. Und die Art und Weise, wie sie handeln, spielt eine entscheidende Rolle bei der Erlangung und Aufrechterhaltung der Demokratie, um Wege zu eröffnen, die Kommunikation ermöglichen und zum Gemeinwohl, dem Recht auf Gesundheit, beitragen.

Habermas (1995) Aus diskursiver Theorie wird die demokratische Willensbildung durch Entscheidungsfindung und kommunikative Annahmen legitimiert, die durch Rationalität die öffentliche Nutzung der Vernunft zum Dialog mit den 
gesundheitlichen Bedürfnissen der Bevölkerung ermöglichen, um faire und kooperative Prozesse zu gewährleisten.

Die gute kooperative Entwicklung, die durch den CIR stattgefunden hat, trägt zur Artikulation und Regulierung von Aktionen innerhalb der Territorien durch die gemeinsamen Verhandlungen bei, die von den föderierten Einheiten in einer solidarischen Perspektive angenommen werden (Lima, 2012; Santos et al., 2014). So kann die Stärkung der Governance in der Gesundheitsregion zusammenbringen, was die Dezentralisierung selbst fragmentiert hat, und die Herausforderungen an die organisierte Versorgungsintegration in Gesundheitsregionen überwinden (LIMA et al., 2016).

Carvalho et al (1995) sind der Ansicht, dass die Gemeinden je nach Bundesverfassung politische und administrative Autonomie bei der Planung und Verwaltung ihrer lokalen Gesundheitssysteme genießen. Daher wäre die einzige Möglichkeit für das Ministerium und das staatliche Gesundheitssekretariat, sich in die kommunale Verwaltung einzumischen, durch Vereinbarungen und Verträge, die zwischen den Parteien frei geschlossen werden.

In den Worten von Noronha et al (2008) ist die SUS "Teil einer breiten Konzeption des Rechts auf Gesundheit und der Rolle des Staates bei der Gewährleistung dieses Rechts, indem sie in ihre institutionelle und Entscheidungsstruktur Räume und Instrumente für die Demokratisierung und die gemeinsame Verwaltung des Gesundheitssystems einbezieht".

Angesichts dieser Merkmale und des verfassungsrechtlich im Gesundheitswesen definierten föderativen Modells ist die Stärkung der geteilten Mittelverwaltung und der Instrumente, die sie begünstigen können, unter ihnen besonders wichtig.

Die Studie ermöglichte es, mehrere Überlegungen über das Thema zu erreichen, ohne die Absicht zu haben, es zu erschöpfen, angesichts der Einschränkungen, die

$\mathrm{RC}: 98473$

Verfügbar in: https://www.nucleodoconhecimento.com.br/technologie-de/regionaleintermanagement 
durch die Forschungskürzung oder sogar durch die Art der von der Studie vorgeschlagenen Analyse auferlegt wurden.

\section{FAZIT}

Um den Zugang zu Gesundheitsdiensten zu gewährleisten und eine umfassende Versorgung zu suchen, ist eine systemische Organisation erforderlich, die aus kooperativen Pakten innerhalb der föderativen Governance besteht, die darauf abzielen, die Organisation von Gesundheitsregionen zu stärken. Das CIR als wichtiges Organ bei der Koordination, Organisation und Konstruktion der Vertragsräume im Hinblick auf den Wiederaufbau und die soziale Organisation zu haben.

Institutionelles Lernen, das Ergebnis der Dynamik der Partizipation am CIR, stärkt das Intermanager-Management und präsentiert sich als wichtiger Baustein für den Aufbau des regionalisierten Netzwerks.

Diese Forschung zeigt, dass die regionale umfassende Planung, die Organisation von Maßnahmen und Dienstleistungen, die Finanzierung und die regionale Governance wesentliche Elemente im Regionalisierungsprozess sind, die durch die Beratungen des CIR erreicht werden, sowie andere Punkte, die in den Sitzungen vereinbart wurden, wie in den Resolutionen beschrieben, mit dem Schwerpunkt auf der Bereitstellung von Gesundheitsdiensten, die der Bevölkerung zur Verfügung stehen.

Die Zusammenarbeit tendiert dazu, die Gemeinden zu begünstigen, die Teil einer Region sind, weil sie die Optimierung der Ressourcen, die solidarische Verwendung der unter den föderierten Einheiten verteilten Mittel und die Garantie der Rentabilität für die Gemeinden mit Teilnahmekapazität ermöglicht, so dass klar ist, dass die CIR / $A L$ ein echter lokaler Raum der Vereinbarung und interföderativen Artikulation sind.

$\mathrm{RC}: 98473$

Verfügbar in: https://www.nucleodoconhecimento.com.br/technologie-de/regionaleintermanagement 


\section{VERWEISE}

AFFONSO, Rui de Brito Álvarez. SILVA, Pedro Luiz Barros. (Org). A federação em perspectiva: ensaios selecionados. São Paulo 10 (3) Edições Fundap; 1995 [acesso em 3 jul 2020]. Disponível em:https://www.seade.gov.br/wpcontent/uploads/2014/07/v10n3.pdf

ANDRADE, Laurielle de Souza.; FRANCISCHETTI, leda. Referência e contrarreferência compreensões e práticas. Sal. \& Transf. Soc. Florianópolis, v. 10, n. 1/2/3, p. 54-63. 2019 [acesso em 13 jul. 2021]. Disponível em: file:///C:/Users/nadja/Dropbox/My\%20PC\%20(LAPTOP-

EA4SVGFV)/Downloads/5281-22616-1-PB.pdf.

BARATA, Luiz Roberto Barradas. TANAKA, Oswaldo Yoshimi. MENDES, José Dínio Vaz. Por um processo de descentralização que consolide os princípios do Sistema Único de Saúde. Epidemiologia e Serviços de Saúde, Brasília, 13 (1) 1524. 2004 [acesso em 02 jun 2020]. Disponível em: http://scielo.iec.gov.br/pdf/ess/v13n1/v13n1a03.pdf

BRASIL. Conselho Nacional de Secretários de Saúde. Legislação Estruturante do SUS/CONASS. Brasília, 2011. 534. [acesso em 25 jul, 2020]. Disponível em: http://bvsms.saude.gov.br/bvs/publicacoes/para_entender_gestao_sus_v13.pdf

BRASIL. Constituição (1988). Constituição da República Federativa do Brasil. promulgada em 5 de outubro de 1988. Brasília, DF: Senado Federal;1988 [acesso $\begin{array}{lllll}\text { em } & 10 & \text { mai } & \text { 2020]. }\end{array}$ https://www2.senado.leg.br/bdsf/bitstream/handle/id/518231/CF88_Livro_EC91_201 6.pdf

BRASIL. Decreto n. 7.508, de 28 de junho de 2011. Regulamenta a Lei n. 8.080, de 19 de setembro de 1990, para dispor sobre a organização do Sistema único de Saúde - SUS, o planejamento da saúde, a assistência à saúde e a articulação

RC: 98473

Verfügbar in: https://www.nucleodoconhecimento.com.br/technologie-de/regionaleintermanagement 
interfederativa, e dá outras providencias. Diário Oficial da União, Poder Executivo, Brasília, DF, 29 jun. 2011. Seção 1, p 1. [acesso em 10 fev 2020]. Disponível em http://www.planalto.gov.br/ccivil_03/_Ato2011-2014/2011/Decreto/D7508.htm.

BRASIL. Lei oํ 8.080, de 19 de setembro de 1990. Lei Orgânica da Saúde. Dispõe sobre as condições para a promoção, proteção e recuperação da saúde, a organização e o funcionamento dos serviços correspondentes e dá outras providências. Brasília, set. 1990. [acesso em 23 abri 2020]. Disponível em: http://www.planalto.gov.br/ccivil_03/leis/18080.htm

BRASIL. Ministério da Saúde. Portaria 4.279, de 30 de dezembro de 2010. Estabelece diretrizes para a organização da Rede de Atenção à Saúde no âmbito do Sistema Único de Saúde (SUS). Diário Oficial da União 31 dez. 2011[acesso em 27 jul 2020].

Disponível

em:

https://bvsms.saude.gov.br/bvs/saudelegis/gm/2010/prt4279_30_12_2010.html

BRASIL. Ministério da Saúde. Secretaria de Atenção à Saúde. Departamento de Regulação, Avaliação e Controle de Sistemas. Diretrizes para a programação pactuada e integrada da assistência à saúde. Brasília. 2006. [acesso em: 27 jul 2020].

Disponível

em: http://bvsms.saude.gov.br/bvs/publicacoes/DiretrizesProgPactuadalntegAssistSaude. pdf

BRASIL. Ministério da Saúde. Secretaria de Gestão e Participativa. Contrato Organizativo da Ação Pública da Saúde. Brasília. 2011 [cesso 30 jul 2020]. Disponível em: http://bvsms.saude.gov.br/bvs/publicacoes/contrato_organizativo_acao_publica_sau de.pdf

RC: 98473

Verfügbar in: https://www.nucleodoconhecimento.com.br/technologie-de/regionaleintermanagement 
BRASIL. Resolução oo 1 setembro de 2011. Gabinete do Ministério da Saúde. Comissão Intergestores Tripartite. Brasília, [citado 07 Jul. 2020]. Disponível em: https://bvsms.saude.gov.br/bvs/saudelegis/cit/2011/res0001_29_09_2011.html

CARVALHO, Guido Ivan. SANTOS, Lenir. Comentários à lei orgânica da saúde: sistema único de saúde. São Paulo: Ed. Hucitec, 1995

CONSELHO DE SECRETÁRIOS MUNICIPAIS DE ALAGOAS. 0 papel dos gestores de saúde nas comissões Intergestores regionais. Maceió, 2014 $\begin{array}{llllll}\text { [acesso } & \mathrm{em} & 12 & \mathrm{abr} & 2020] & \text { Disponível em: }\end{array}$ http://www.cosemsal.org/old/public/documentos/manual-azul-o-papel-dos-gestoresde-saude.pdf

CURVINA, Ana Cristina Carvalho. A regionalização da saúde no federalismo brasileiro. Cad. Ibero-Amer. Dir. Sanit., Brasília, 6(2):42-57, abr./jun, 2017. [acesso em 13 fev 2020]. Disponível em: http://dx.doi.org/10.17566/ciads.v6i2.385

DOURADO, Daniel de Araújo.; ELIAS, Paulo Eduardo Mangeon. Regionalização e dinâmica política do federalismo sanitário brasileiro. Rev. Saúde Pública. São Paulo, v. 45, n. 1, p. 204-11. 2011. [acesso em 05 jul. 2021]. Disponível em: https://www.scielo.br/j/rsp/a/bCWB3sWTXQLXWjjFkcWwgLt/?format=pdf\&lang=pt

DUARTE, Ligia Schiavon.; MENDES, Áquilas Nogueira.; LOUVISONA, Marília, Cristina Prado. O processo de regionalização do SUS e a autonomia municipal no uso dos recursos financeiros: uma análise do estado de São Paulo (2009-2014). Saúde Debate, Rio de Janeiro, v. 42, n. 116, p. 25-37. 2018. [acesso em 10 jul 2020].

https://www.scielo.br/j/sdeb/a/gcPyhjjWdrqZcG9V4nYhPBd/?format=pdf\&lang=pt.

HABERMAS, Jurgen. Três Modelos Normativos de Democracia. Cadernos da Escola do Legislativo, Belo Horizonte, 3 (3)105-122, jan.-jun., 1995.

RC: 98473

Verfügbar in: https://www.nucleodoconhecimento.com.br/technologie-de/regionaleintermanagement 
LEVCOVITZ, Eduardo. LIMA, Luciana Dias de. MACHADO, Cristiane. Vieira. Política de saúde nos anos 90: relações intergovernamentais e o papel das Normas Operacionais Básica. Ciência e Saúde Coletiva, Rio de Janeiro, 6, (2) 269-291, 2001 [acesso em 10 jul 2020]. Disponível em: https://www.scielo.br/pdf/csc/v6n2/7003.pdf.

LIMA, Luciana Dias de. et al. Regionalização e acesso à saúde nos estados brasileiros: condicionantes históricos e político-institucionais. Ciência e Saúde Coletiva, Rio de Janeiro, 17 (11) 2881-2892, 2012 [acesso 10 jun 2020]. Disponível em: https://www.scielo.br/pdf/csc/v17n11/v17n11a04.pdf

LIMA, Luciana Dias de.; ALBUQUERQUE, Mariana Vercesi.; SCATENA, João Henrique Gurtler. Quem governa e como se governam as regiões e redes de atenção à saúde no Brasil? Contribuições para o estudo da governança regional na saúde. Novos Caminhos, n. 8. 2016. [acesso em 29 fev 2020]. Disponível: https://www.resbr.net.br/wp-content/uploads/2016/02/Novos-Caminhos-8.pdf.

LIMA, Luciana Dias de. et al. Descentralização e regionalização: dinâmica e condicionamento da implantação do pacto pela saúde no Brasil. Ciência \& Saúde Coletiva, n. 17, v. 7, p. 1903-1914, 2012. [acesso em 29 fev. 2020]. Disponível em: https://www.scielo.br/j/csc/a/FyqLwWmbv8WQs8BPW8scpTt/?lang=pt

MENICUCCI, Telma Maria Gonçalves. Regionalização no federalismo brasileiro. Cad. Saúde Pública 2019; 35 Sup 2: e00078419. [acesso em 29 fev 2020]. Disponível: https://www.scielosp.org/article/csp/2019.v35suppl2/e00078419/pt/

MINAYO, Maria Cecília de Souza. O desafio do conhecimento. Pesquisa qualitativa em saúde; $12^{\underline{a}}$ ed; São Paulo. Hucitec. 2010. 407p. ISBN 978-85-271-0181-3.

MOREIRA, Laura Monteiro de.; FERRÉ, Felipe.; ANDRADE, Eli lola Gurgel. Financiamento, descentralização e regionalização: transferências federais e as redes de atenção em Minas Gerais, Brasil. Ciência \& Saúde Coletiva, n 22, v. 4, p.

$\mathrm{RC}: 98473$

Verfügbar in: https://www.nucleodoconhecimento.com.br/technologie-de/regionaleintermanagement 
1245-1256, 2017. [acesso em 21 ago 2021]. Disponível em: https://www.scielosp.org/pdf/csc/2017.v22n4/1245-1256/pt.

NORONHA, José Carvalho de. LIMA, Luciana. Dias de. MACHADO, Cristiane Vieira. O Sistema Único de Saúde: SUS. In: Giovanella L. et al. (Org.). Políticas e sistemas de saúde no Brasil. Rio de Janeiro: Fiocruz, 2008. p. 365-394.

PINAFO, Elisangela. et al. Problemas e estratégias de gestão do SUS: a vulnerabilidade dos municípios de pequeno porte. Ciência \& Saúde Coletiva, n2. 25, v. 5, p. 1619-1628, 2020 [acesso em 21 ago 2021]. Disponível em: https://scielosp.org/pdf/csc/2020.v25n5/1619-1628/pt

SANTOS, Adriano Maia dos. GIOVANELLA, Ligia. Governança regional: estratégias e disputas para gestão em saúde. Revista de Saúde Pública, São Paulo, 48 (4) 622-631, 2014[acesso em 10 jun 2020]. Disponível em: https://www.resbr.net.br/wpcontent/uploads/historico/governan\%C3\%A7a_regional.pdf

SANTOS, Lenir. CAMPOS, Gastão Wagner de Souza. SUS Brasil: a região de saúde como caminho saúde e Sociedade, São Paulo, 24 (2) 438-446, 2015 [acesso em 10 jul 2020]. Disponível em: https://www.scielo.br/pdf/sausoc/v24n2/0104-1290-sausoc-24-02-00438.pdf

SANTOS, Lenir. Região de saúde e suas redes de atenção: modelo organizativosistêmico do SUS. Ciência \& Saúde Coletiva, 22(4):1281-1289, 2017. [acesso em 29 jul, 2020]. Disponível em: https://www.scielosp.org/pdf/csc/2017.v22n4/1281$1289 / \mathrm{pt}$

SOARES, Marcia Miranda. MACHADO, José Ângelo. Federalismo e políticas públicas. Brasília: Enap, 2018. 112. [acesso em: 29 fev 2020]. ISBN: 978-85-2560081-3Disponível em: https://repositorio.enap.gov.br/bitstream/1/3331/1/Livro_Federalismo\%20e\%20Pol\% C3\%ADticas\%20P\%C3\%BAblicas.pdf.

$\mathrm{RC}: 98473$

Verfügbar in: https://www.nucleodoconhecimento.com.br/technologie-de/regionaleintermanagement 
VIANA, Ana Luiza D’Ávila. LIMA, Luciana Dias. OLIVEIRA, Roberta. Gondim de. Descentralização e federalismo: a política de saúde em novo contexto - lições do caso brasileiro. Ciência e Saúde coletiva. 2002 Jul; 7 (3):493-507. [acesso em 5 mai 2020]. Disponível em: https://www.scielo.br/pdf/csc/v7n3/13027.pdf

VIANA, Ana Luiza D' Ávila. et al. Novas Perspectivas para a regionalização da saúde; São Paulo em perspectiva, 22 (1) 92-106, jan/jun.2008 [acesso em 7 jun 2020].

http://produtos.seade.gov.br/produtos/spp/v22n01/v22n01_07.pdf

WAGNER, Gastão. Efeitos paradoxais da descentralização do Sistema Único de Saúde do Brasil; In: Fleury S. (Org.). Democracia, descentralização e desenvo/vimento: Brasil e Espanha. Rio de Janeiro: FGV, 2006. 417-442. Disponível em https://www.scielo.br/pdf/csc/v17n11/v17n11a04.pdf.

Gepostet: März 2021.

Genehmigt: September 2021.

RC: 98473

Verfügbar in: https://www.nucleodoconhecimento.com.br/technologie-de/regionaleintermanagement 